\title{
Forward-Integration Riccati-Based Output-Feedback Control of Linear Time-Varying Systems
}

\author{
Avishai Weiss, Ilya Kolmanovsky, and Dennis S. Bernstein
}

\begin{abstract}
In applications involving time-varying systems, the state dynamics matrix is often not known in advance. To address this problem, this paper investigates the effectiveness of a forward-in-time Riccati-based control law. This approach is motivated by the fact that the optimal state estimator is based on a forward-in-time Riccati-based solution that does not require advance knowledge of the system dynamics. In this paper we show that a forward-in-time Riccati-based control law stabilizes the system if the dynamics of the quasi-dual system are asymptotically stable. This property holds if the closedloop dynamics are symmetric, and, for some plants, is achieved by dynamics with sufficiently fast time variation. In addition, using a separation principal type result, we guarantee closedloop stability in the case of output feedback.
\end{abstract}

\section{INTRODUCTION}

A bedrock principle of dynamic programming is the fact that optimal paths are determined by a backwards-intime procedure. This procedure gives rise to the Bellman equation, which in turn is related to the Hamilton-JacobiBellman partial differential equation [1]. Within the context of optimal control, the Bellman equation provides optimal control histories over a specified control horizon. In the special case of the linear system

$$
\dot{x}(t)=A(t) x(t)+B(t) u(t)
$$

with the quadratic cost

$$
J(u)=\int_{0}^{t_{\mathrm{f}}} x^{\mathrm{T}}(t) R_{1} x(t)+u^{\mathrm{T}}(t) R_{2} u(t) \mathrm{d} t
$$

the backwards nature of the optimal control law

$$
u(t)=-R_{2}^{-1} B^{\mathrm{T}}(t) P_{\mathrm{b}}(t) x(t)
$$

is evident from the fact that the solution $P_{\mathrm{b}}(t)$ of the control Riccati equation

$$
\begin{aligned}
-\dot{P}_{\mathrm{b}}(t)= & A^{\mathrm{T}}(t) P_{\mathrm{b}}(t)+P_{\mathrm{b}}(t) A(t) \\
& -P_{\mathrm{b}}(t) B(t) R_{2}^{-1} B^{\mathrm{T}}(t) P_{\mathrm{b}}(t)+R_{1}
\end{aligned}
$$

is obtained by integrating backwards from the boundary condition $P_{\mathrm{b}}\left(t_{\mathrm{f}}\right) \geq 0$ specified at the final time $t_{\mathrm{f}}$. This procedure yields the closed-loop dynamics

$$
\dot{x}(t)=A_{\mathrm{c}, \mathrm{b}}(t) x(t),
$$

where $A_{\mathrm{c}, \mathrm{b}}(t) \triangleq A(t)-B(t) R_{2}^{-1} B^{\mathrm{T}}(t) P_{\mathrm{b}}(t)$.

In practice, a backwards-in-time solution procedure has the significant drawback that the dynamics of the system, that is, the matrices $A(t)$ and $B(t)$, must be known in advance.

Department of Aerospace Engineering, University of Michigan, Ann Arbor, MI 48109 USA
However, in many applications, the system trajectory, and thus the linearized dynamics, are not known in advance. Consequently, there is interest in forward-in-time solution procedures, in particular, for applications of model predictive and receding horizon control [2], [3], [4].

The dual of the control problem is the state estimation problem involving the observed dynamics

$$
\begin{aligned}
\dot{x}(t) & =A(t) x(t)+w_{1}(t), \\
y(t) & =C(t) x(t)+w_{2}(t) .
\end{aligned}
$$

For this problem, the estimator Riccati equation

$$
\begin{aligned}
\dot{Q}(t)= & A(t) Q(t)+Q(t) A^{\mathrm{T}}(t) \\
& -Q(t) C^{\mathrm{T}}(t) V_{2}^{-1} C(t) Q(t)+V_{1}
\end{aligned}
$$

is solved forward in time with the initial-time boundary condition $Q\left(t_{0}\right) \geq 0$ and is guaranteed to minimize the state estimation cost. The matrices $V_{1}$ and $V_{2}$ represent the covariances of the process noise $w_{1}$ and sensor noise $w_{2}$, respectively. Under uniform complete reconstructability conditions the resulting closed-loop error system

$$
\dot{e}(t)=A_{\mathrm{e}}(t) e(t),
$$

where $A_{\mathrm{e}}(t) \triangleq A(t)-Q(t) C^{\mathrm{T}}(t) V_{2}^{-1} C(t)$, is exponentially stable [1]. Note that, unlike the case of the control Riccati equation (4), implementation of the optimal state estimator in terms of the estimator Riccati equation (8) does not require advance knowledge of the dynamics matrices $A(t)$ and $C(t)$.

It is, therefore, natural to ask whether a dual version of the forward-in-time estimator Riccati equation (8) can provide a suboptimal, forward-in-time control law. In particular, we consider the control law

$$
u(t)=-R_{2}^{-1} B^{\mathrm{T}}(t) P_{\mathrm{f}}(t) x(t),
$$

where $P_{\mathrm{f}}(t)$ is the solution to the forward-in-time control Riccati equation

$$
\begin{aligned}
\dot{P}_{\mathrm{f}}(t)= & A^{\mathrm{T}}(t) P_{\mathrm{f}}(t)+P_{\mathrm{f}}(t) A(t) \\
& -P_{\mathrm{f}}(t) B(t) R_{2}^{-1} B^{\mathrm{T}}(t) P_{\mathrm{f}}(t)+R_{1},
\end{aligned}
$$

with the initial-time boundary condition $P_{\mathrm{f}}\left(t_{0}\right) \geq 0$. Using this control law, the closed-loop dynamics are given by

$$
\dot{x}(t)=A_{\mathrm{c}, \mathrm{f}}(t) x(t),
$$

where $A_{\mathrm{c}, \mathrm{f}}(t) \triangleq A(t)-B(t) R_{2}^{-1} B^{\mathrm{T}}(t) P_{\mathrm{f}}(t)$. Note that (11) differs from the standard backwards-in-time Riccati equation (4) by the absence of the minus sign on the left-hand 
side; this feature along with the specification of an initialtime boundary condition in place of a final-time boundary condition, render it closer in spirit to the estimator Riccati equation (8) than the backwards-in-time Riccati equation (4). In fact, (11) is equivalent to the estimator Riccati equation with $A(t), C(t), V_{1}$, and $V_{2}$ replaced by the dual variables $A^{\mathrm{T}}(t), B^{\mathrm{T}}(t), R_{1}$, and $R_{2}$. Furthermore, $A_{\mathrm{c}, \mathrm{f}}(t)=A_{\mathrm{e}}^{\mathrm{T}}(t)$ with the appropriate substitutions.

Thus, assuming that (9) is asymptotically stable, it remains to be determined whether the transposed system

$$
\dot{e}(t)=A_{\mathrm{e}}^{\mathrm{T}}(t) e(t)
$$

is also asymptotically stable. We call (13) the quasi-dual of (9) to distinguish it from the "true" dual (5) of (9). Variations of the backwards-in-time Riccati equation are discussed in [5], although that paper does not consider the use of quasidual dynamics.

The above discussion reflects the development in [6], [7] of forward-Riccati-based full-state-feedback control laws for time-varying systems. The approach taken in [6], [7] involves the definition of a state $z(t)$ whose magnitude is inversely proportional to the magnitude of $x(t)$ and whose dynamics are obtained by a time-dependent similarity transformation of the dynamics of $x(t)$. Consequently, the forward-Riccatibased controller for the system with state $z(t)$ anti-stabilizes the dynamics of $z(t)$; consequently, the original dynamics involving $x(t)$ are stabilized. This approach has the advantage over the backwards Riccati solution mentioned above, namely, the time-varying matrices $A(t)$ and $B(t)$ need not be known in advance.

Since the dynamics for $z(t)$ depend on a state-dependent transformation of the dynamics of the state $x(t)$, the controller derived in [6], [7] is confined to full-state feedback. The development of our feedback law (10), (11) maintains a linear form without resorting to nonlinear transformations. Therefore, one of the main contributions of the present paper is to develop a forward-Riccati-based control law that does not require knowledge of the full state and thus is applicable to output feedback. In particular, by removing the requirement that the full state be known, we construct an observer-based compensator that uses the (forward) Riccati equation of the full-state estimator (8) ,and use a separation principle type result to guarantee closed-loop stability. In this way, we construct an output-feedback control law for timevarying systems whose dynamics matrices are not known in advance. This output feedback controller is described in Section V.

The goal of the present paper is thus to investigate the applicability of the forward-in-time Riccati equation (11) to time-varying systems in which $A(t)$ and $B(t)$ are not known in advance. Clearly, the forward-in-time controller is stabilizing if the quasi-dual system (13) is asymptotically stable. In the time-invariant case, a matrix $A$ is Hurwitz if and only if its transpose $A^{\mathrm{T}}$ is Hurwitz. Perhaps somewhat surprisingly, the same cannot be concluded in the timevarying case. In other words, stability of a time-varying system does not guarantee stability of the system with the transposed dynamics. As an illustrative example, consider

$$
A(t)=\left[\begin{array}{cc}
-1 & \sin (t)-1 \\
\sin (t)+1 & \cos (t)
\end{array}\right],
$$

and its transpose

$$
A^{\mathrm{T}}(t)=\left[\begin{array}{cc}
-1 & \sin (t)+1 \\
\sin (t)-1 & \cos (t)
\end{array}\right] .
$$

Since $A(t)$ is periodic, stability depends on the characteristic multipliers, that is, the eigenvalues of the monodromy matrix $\Phi(T, 0)$, where $\Phi$ denotes the state transition matrix. Figure 1 shows that while $A(t)$ is stable as seen by its state trajectories and characteristic multipliers, its transpose is not.

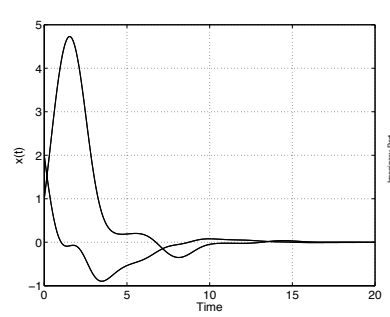

(a) State trajectories for $A(t)$



(c) State trajectory for $A^{\mathrm{T}}(t)$

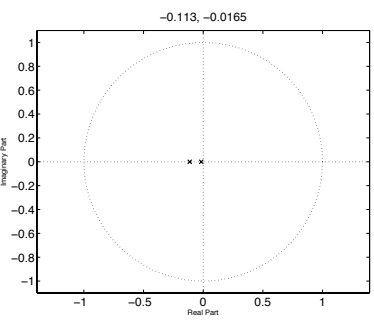

(b) Characteristic Multipliers

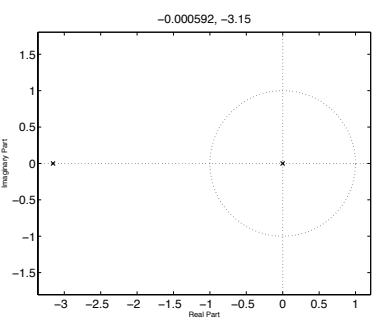

(d) Characteristic Multipliers
Fig. 1: (a), (b): State trajectories and characteristic multipliers for $A(t)$. (c), (d): State trajectories and characteristic multipliers for $A^{\mathrm{T}}(t)$.

The main contribution of the present paper is a collection of results under which the forward Riccati-based controller (10), (11) is stabilizing. In particular, we demonstrate that symmetry of the closed-loop dynamics is a sufficient condition for closed-loop stability. This property is demonstrated by the quasi-duality between the state estimator and the forward-Riccati-based controller, and is also brought out through Lyapunov analysis. In addition, we show that some plants are stabilizable only when the time-varying dynamics are of sufficiently high frequency. This property is demonstrated through averaging theory. We also demonstrate using a separation principle type result for linear time-varying systems, that stability is retained in the output feedback case. Finally, experience with numerous numerical examples suggests that the forward Riccati output feedback approach is stabilizing in a much broader set of situations.

The paper is organized as follows. Section II uses Lyapunov analysis to show stability with the forward-in-time 
controller when the closed-loop system has certain symmetric properties. In Section III we examine the properties of the state transition matrix and present additional conditions under which the forward-in-time controller is stabilizing. In Section IV we demonstrate that the forward-in-time controller is stabilizing when the frequency of the time variation is sufficiently high. In Section $\mathrm{V}$ we show that we have separation of the regulator and estimator dynamics under output feedback. In Section VI we provide illustrative numerical examples that highlight the effectiveness and desirability of the forward-in-time control law. Section VII presents an alternate forward-in-time Riccati equation which results in a stabilizing controller for scarlar systems, and, in special cases, for non-scalar systems. Finally, Section VIII makes concluding remarks.

\section{LYAPUNOV ANALYSIS}

We determine sufficient conditions for stability of the forward-in-time controller. We use Lyapunov analysis to examine the stability of the closed-loop system (12).

However, since (12) is the quasi-dual of (9), we first look at the closed-loop estimation system (9) to gain insight. Let $\bar{\Sigma}(t)=C^{\mathrm{T}}(t) V_{2}^{-1} C(t), Q\left(t_{0}\right)>0$, and rewrite (8) as

$$
\dot{Q}(t)=A_{\mathrm{e}}(t) Q(t)+Q(t) A_{\mathrm{e}}^{\mathrm{T}}(t)+Q(t) \bar{\Sigma}(t) Q(t)+V_{1} .
$$

Let $V(e, t)=e^{\mathrm{T}} Q^{-1}(t) e$ as in [9]. Then,

$$
\begin{aligned}
\dot{V} & =\dot{e}^{T} Q^{-1}(t) e+e^{T} Q^{-1}(t) \dot{e}+e^{T} \dot{Q}^{-1}(t) e \\
& =-e^{T}\left(\bar{\Sigma}(t)+Q^{-1}(t) V_{1} Q^{-1}(t)\right) e<0 .
\end{aligned}
$$

Similarly, we let $\Sigma(t)=B(t) R_{2}^{-1} B^{\mathrm{T}}(t), P_{\mathrm{f}}\left(t_{0}\right)>0$ and rewrite (11) as

$\dot{P}_{\mathrm{f}}(t)=A_{\mathrm{c}, \mathrm{f}}^{\mathrm{T}}(t) P_{\mathrm{f}}(t)+P_{\mathrm{f}}(t) A_{\mathrm{c}, \mathrm{f}}(t)+P_{\mathrm{f}}(t) \Sigma(t) P_{\mathrm{f}}(t)+R_{1}$.

Let $V(x, t)=x^{\mathrm{T}} P_{\mathrm{f}}^{-1}(t) x$. Then,

$$
\begin{aligned}
\dot{V}= & \dot{x}^{T} P_{\mathrm{f}}^{-1}(t) x+x^{T} P_{\mathrm{f}}^{-1}(t) \dot{x}+x^{T} \dot{P}_{\mathrm{f}}^{-1}(t) x \\
= & -x^{\mathrm{T}}\left(\Sigma(t)+P_{\mathrm{f}}^{-1}(t) R_{1} P_{\mathrm{f}}^{-1}(t)\right) x \\
& +x^{\mathrm{T}}\left(P_{\mathrm{f}}^{-1}(t)\left(A_{\mathrm{c}, \mathrm{f}}(t)-A_{\mathrm{c}, \mathrm{f}}^{\mathrm{T}}(t)\right)\right. \\
& \left.+\left(A_{\mathrm{c}, \mathrm{f}}^{\mathrm{T}}(t)-A_{\mathrm{c}, \mathrm{f}}(t)\right) P_{\mathrm{f}}^{-1}(t)\right) x .
\end{aligned}
$$

Note the extra term in this expression versus (14). Thus, a sufficient condition for asymptotic stability of (12) is that $A_{\mathrm{c}, \mathrm{f}}(t)$ is symmetric. As an example, this condition holds if $A(t)$ is symmetric, $B(t)=G(t) D(t)$, where $G(t)$ is an orthogonal matrix, $D(t)=f(t) I$ is a constant matrix, and $R_{1}=R_{2}=\rho I$.

\section{STATE TRANSITION MATRIX ANALYSIS}

The state transition formula for the closed-loop system (12) is given by the Peano-Baker series

$$
\begin{aligned}
\Phi\left(t, t_{0}\right)= & I+\int_{t_{0}}^{t} A_{\mathrm{c}, \mathrm{f}}\left(\sigma_{1}\right) d \sigma_{1} \\
& +\int_{t_{0}}^{t} A_{\mathrm{c}, \mathrm{f}}\left(\sigma_{1}\right) \int_{t_{0}}^{\sigma_{1}} A_{\mathrm{c}, \mathrm{f}}\left(\sigma_{2}\right) d \sigma_{2} d \sigma_{1} \ldots
\end{aligned}
$$

It follows from (15) that, if $A_{\mathrm{c}, \mathrm{f}}(t)$ is symmetric, then the state transition matrix for $A_{\mathrm{c}, \mathrm{f}}(t)$ is equal to the state transition matrix for $A_{\mathrm{c}, \mathrm{f}}^{\mathrm{T}}(t)$.

Suppose next that $A_{\mathrm{c}, \mathrm{f}}(t)$ and its integral commute, that is,

$$
A_{\mathrm{c}, \mathrm{f}}(t) \int_{t_{0}}^{t} A_{\mathrm{c}, \mathrm{f}}(\sigma) d \sigma=\int_{t_{0}}^{t} A_{\mathrm{c}, \mathrm{f}}(\sigma) d \sigma A_{\mathrm{c}, \mathrm{f}}(t) \quad \forall t,
$$

or equivalently [8], if

$$
A_{\mathrm{c}, \mathrm{f}}\left(t_{1}\right) A_{\mathrm{c}, \mathrm{f}}\left(t_{2}\right)=A_{\mathrm{c}, \mathrm{f}}\left(t_{2}\right) A_{\mathrm{c}, \mathrm{f}}\left(t_{1}\right) \quad \forall t_{1}, t_{2} .
$$

Then, the Peano-Baker series simplifies to

$$
\Phi\left(t, t_{0}\right)=\exp \left[\int_{t_{0}}^{t} A_{\mathrm{c}, \mathrm{f}}(\sigma) d \sigma\right]
$$

while

$$
\Phi^{\mathrm{T}}\left(t, t_{0}\right)=\exp \left[\int_{t_{0}}^{t} A_{\mathrm{c}, \mathrm{f}}^{\mathrm{T}}(\sigma) d \sigma\right]
$$

is the state transition matrix for $A_{\mathrm{c}, \mathrm{f}}^{\mathrm{T}}(t)$. In this case, stability of $A_{\mathrm{c}, \mathrm{f}}(t)$ is equivalent to stability of $A_{\mathrm{c}, \mathrm{f}}^{\mathrm{T}}(t)$.

It thus follows that if either $A(t)$ is symmetric for all $t$, or if $A\left(t_{1}\right)$ and $A\left(t_{2}\right)$ commute for all $t_{1}$ and $t_{2}$, then, the stability of the closed-loop regulator sytem (12) is equivalent to the stability of the closed-loop estimator error system (9). Therefore, in either of these cases it follows that the forwardin-time controller (10) is guaranteed to stabilize (1).

\section{AVERAGING ANALYSIS}

We now analyze the stability of the closed-loop system with the forward-in-time controller (10) at high frequencies. Specifically, we demonstrate that the forward-in-time controller is stabilizing under sufficiently fast time variation in the closed-loop dynamics.

Define the time-invariant matrices $\bar{A}$ and $\bar{B}$ by

$$
\begin{aligned}
\bar{A} & \triangleq \frac{1}{T} \int_{0}^{\mathrm{T}} A(\omega t) d t \\
\bar{B} \bar{B}^{\mathrm{T}} & \triangleq \frac{1}{T} \int_{0}^{\mathrm{T}} B(\omega t) R_{2}^{-1} B^{\mathrm{T}}(\omega t) d t .
\end{aligned}
$$

Theorem 1: Replace $A(t)$ and $B(t)$ in (1) by timeperiodic $A(\omega t)$ and $B(\omega t)$ with period $T=2 \pi / \omega$, where $\omega$ is a frequency parameter. Apply the forward-in-time control (10) with $P_{\mathrm{f}}(t)$ as given in (11). Suppose $(\bar{A}, \bar{B})$ is stabilizable, $\left(\bar{A}, R_{1}\right)$ is detectable, and let $P_{\mathrm{f}}(0) \geq 0$. Then, there exists a frequency $\omega^{*}$ such that, for all $\omega>\omega^{*}, P_{\mathrm{f}}(t)$ has an exponentially stable periodic solution of period $T$ in an $O(\epsilon)$ neighborhood of $P^{*}$, where $P^{*}$ is a positive-semidefinite solution of the algebraic Riccati equation,

$$
0=\bar{A}^{\mathrm{T}} P^{*}+P^{*} \bar{A}-P^{*} \bar{B} R_{2}^{-1} \bar{B}^{\mathrm{T}} P^{*}+R_{1},
$$

and $x(t) \rightarrow 0$ as $t \rightarrow \infty$. 
Proof: Define the scaled time $\tau \triangleq \omega t$, and small parameter $\epsilon \triangleq \frac{1}{\omega}$. Rewrite (11) and (12) as

$$
\begin{aligned}
\frac{d}{d \tau} P_{\mathrm{f}}(\tau)= & \epsilon\left(A^{\mathrm{T}}(\tau) P_{\mathrm{f}}(\tau)+P_{\mathrm{f}}(\tau) A(\tau)\right. \\
& \left.-P_{\mathrm{f}}(\tau) B(\tau) R_{2}^{-1} B^{\mathrm{T}}(\tau) P_{\mathrm{f}}(\tau)+R_{1}\right), \\
\frac{d}{d \tau} x(\tau)= & \epsilon\left(A(\tau)-B(\tau) R_{2}^{-1} B^{\mathrm{T}}(\tau) P_{\mathrm{f}}(\tau)\right) x(\tau) .
\end{aligned}
$$

Then, averaging equations (23) and (24), we obtain the timeinvariant averaged system

$$
\begin{aligned}
\dot{\bar{P}}(t) & =\bar{A}^{\mathrm{T}} \bar{P}(t)+\bar{P}(t) \bar{A}-\bar{P}(t) \bar{B} \bar{B}^{\mathrm{T}} \bar{P}(t)+R_{1}, \\
\dot{\bar{x}}(t) & =\left(\bar{A}-\bar{B} \bar{B}^{\mathrm{T}} \bar{P}(t)\right) \bar{x}(t) .
\end{aligned}
$$

Applying Theorems 4.11 and 3.7 in [1] to (25) and (26) with the appropriate dual substitutions yields $\bar{P}(t) \rightarrow P^{*}$ and $\bar{x}(t) \rightarrow 0$ as $t \rightarrow \infty$. Furthermore, under the assumption that the Hamiltonian matrix

$$
H=\left[\begin{array}{cc}
\bar{A} & -\bar{B} \bar{B}^{\mathrm{T}} \\
-R_{1} & -\bar{A}^{\mathrm{T}}
\end{array}\right]
$$

has no eigenvalues on the imaginary axis, the convergence is exponential [10].

Then, applying Theorem 8.3 in [11] for $\omega>\omega^{*}$, where $\omega^{*}$ is sufficiently large, renders the solution of the time-varying Riccati equation (11) exponentially convergent to a periodic solution in an $O(\epsilon)$ neighborhood of $P^{*}$.

We complete the proof by noting that stability of (26) implies that there exists a positive-definite matrix $M$ such that

$$
\left(\bar{A}-\bar{B} \bar{B}^{\mathrm{T}} P^{*}\right)^{\mathrm{T}} M+M\left(\bar{A}-\bar{B} \bar{B}^{\mathrm{T}} P^{*}\right)=-\epsilon I<0 .
$$

For the original closed-loop system (12), assuming $\omega^{*}$ sufficiently large, the same matrix $M$ yields

$$
\begin{aligned}
& \left(A(t)-B(t) R_{2}^{-1} B^{\mathrm{T}}(t) P_{\mathrm{f}}(t)\right)^{\mathrm{T}} M \\
& \quad+M\left(A(t)-B(t) R_{2}^{-1} B^{\mathrm{T}}(t) P_{\mathrm{f}}(t)\right)=-\frac{\epsilon}{2} I<0 .
\end{aligned}
$$

Therefore $x(t) \rightarrow 0$ as $t \rightarrow \infty$.

\section{OUTPUT FEEDBACK}

We now consider the situation where the full-state measurement is not available. We propose the observer-based dynamic compensator

$$
\begin{aligned}
\dot{\hat{x}}(t)= & A(t) \hat{x}(t)+B(t) u(t) \\
& +F(t)(y(t)-C(t) \hat{x}(t)), \\
u(t)= & -R_{2}^{-1} B^{\mathrm{T}}(t) P_{\mathrm{f}}(t) \hat{x}(t),
\end{aligned}
$$

where $F(t)=Q(t) C^{\mathrm{T}}(t) V_{2}^{-1}$ is produced using the estimator Riccati equation (8). Note that, unlike the standard LQG problem, the entire system of differential equations is solved forward-in-time, and therefore (27), (28) can be implemented on a time-varying system without full-state feedback and without knowing the dynamics $A(t), B(t)$, and $C(t)$ in advance.
The closed loop system consisting of the observer-based dynamic compensator (28) interconnected with the linear system (1) can be described by the linear system of dimension $2 n$

$$
\left[\begin{array}{c}
\dot{x}(t) \\
\dot{\hat{x}}(t)
\end{array}\right]=A_{\mathrm{cl}}(t)\left[\begin{array}{c}
x(t) \\
\hat{x}(t)
\end{array}\right],
$$

where $n$ is the dimension of the state $x(t)$ and where

$$
A_{\mathrm{cl}}(t) \triangleq\left[\begin{array}{cc}
A(t) & B(t) K(t) \\
F(t) C(t) & A(t)-F(t) C(t)+B(t) K(t)
\end{array}\right],
$$

where $K(t)=-R_{2}^{-1} B^{\mathrm{T}}(t) P_{f}(t)$ and the Kalman gain $F(t)$ is given in (27).

We now show, under appropriate assumptions, the closedloop system (29) is uniformly exponentially stable if both the regulator and estimator dynamics are uniformly exponentially stable.

First, we restate Theorem 4.9 of [1]. Suppose that $A(t)$ is continuous and bounded, $B(t)$ is piecewise continuous and bounded, and that $R_{1}, R_{2} \geq \alpha I$ for $\alpha>0$. Let $P_{f}(t)$ be a solution of the Riccati equation (11) with initial condition $P_{f}\left(t_{0}\right) \geq 0$. Then, if the pair $\left(A^{\mathrm{T}}(t), B^{\mathrm{T}}(t)\right)$ is uniformly completely reconstructable, there exists a solution of the Riccati equation (11) $\bar{P}_{f}(t)$ such that for any $P_{f}\left(t_{0}\right) \geq 0$, $P_{f}(t)-\bar{P}_{f}(t) \rightarrow 0$ as $t \rightarrow \infty$. Furthermore, $\bar{P}_{f}(t)$ has an upper bound, and is nonnegative-definite for all $t$.

Now, the following mirrors Theorem 15.5 of [8].

Theorem 2: Let the assumptions of Theorem 4.9 of [1] as stated above hold, let the pair $(A(t), C(t))$ be uniformly completely reconstructable, and define $\delta \triangleq\left\|R_{2}^{-1}\right\|$. Additionally, suppose there exist positive constants $\varepsilon_{1}, \varepsilon_{2}, \beta_{1}$, and $\beta_{2}$ such that

$$
\varepsilon_{1} I \leq P_{f}(t) \leq \varepsilon_{2} I,
$$

and

$$
\int_{\tau}^{t}\|B(\sigma)\|^{2} d \sigma \leq \beta_{1}+\beta_{2}(t-\tau) .
$$

Finally, assume that the forward-in-time controller (10) is exponentially stabilizing. Then, the closed-loop system (29) is uniformly exponentially stable.

The proof is omitted due to space constraints. This result implies that we obtain separation of the regulator and estimator dynamics in the sense that that if both the regulator and estimator dynamics are uniformly exponentially stable, the closed-loop system (29) is uniformly exponentially stable.

\section{ILLUSTRATIVE EXAMPLES}

Consider a mass attached to a wall via a spring with variable stiffness $k(t)$, as shown in Fig. 2.

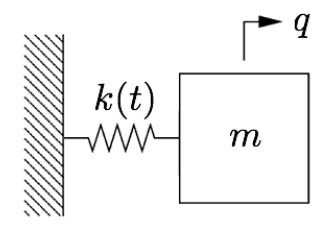


Fig. 2: A mass constrained by a spring with time-varying stiffness.

The open-loop system is described by

$x(t)=\left[\begin{array}{c}q \\ \dot{q}\end{array}\right], \quad A(t)=\left[\begin{array}{cc}0 & 1 \\ \frac{-k(t)}{m} & 0\end{array}\right], \quad B(t)=\left[\begin{array}{c}0 \\ \frac{1}{m}\end{array}\right]$,

where $k(t)$ is a time-varying stiffness that may assume positive or negative values. Let $k(t)=\sin (t), m=1$ $q(0)=2$, and $\dot{q}(0)=1$. Fig. 3 shows the open-loop response, which is unstable. We wish to stabilize this system.

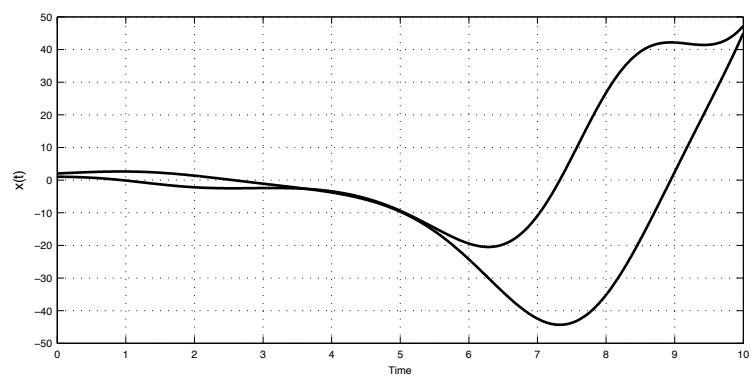

Fig. 3: Open-loop response.

First we assume that $k(t)$ is known in advance. We apply the backwards-in-time optimal control (3) over a finite interval of 10 seconds. Let $R_{1}=R_{2}=I$ and $P_{\mathrm{b}}(10)=0$. Fig. 4a shows the state trajectories and control action.

Next we assume that $k(t)$ is not known ahead of time. Instead, a perfect measurement of $k(t)$ is available at time $t$. We let $R_{1}=R_{2}=I, P_{\mathrm{f}}(0)=0$, and apply the forwardin-time control (10). Fig. $4 \mathrm{~b}$ shows the state trajectories and control action. Both backwards-in-time and forward-in-time control laws stabilize the system to the origin within 10 seconds.
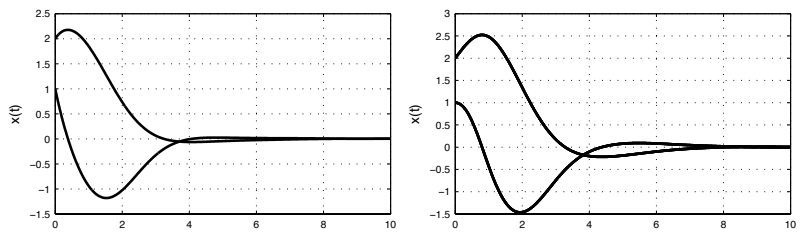

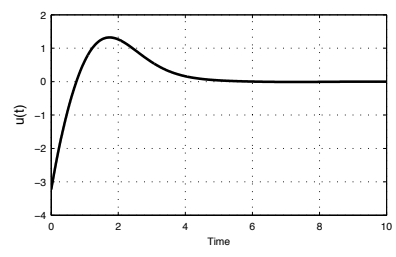

(a) Backwards-in-time control

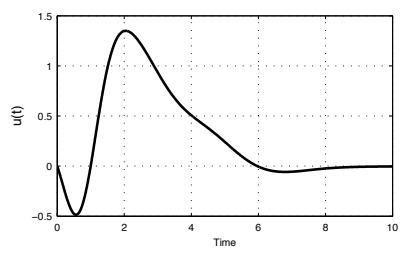

(b) Forward-in-time control
Fig. 4: Full-state feedback for the mass-spring example.

Next we consider the case where the full-state measurement is not available. In this case, we use dynamic output feedback (27), (28) to stabilize the system. Suppose that

$$
C(t)=\left[\begin{array}{ll}
1 & 0
\end{array}\right],
$$

so that only the position of the mass is measured. Let $\hat{x}(t)=$ $0, V_{1}=V_{2}=I$, and $Q(0)=0$. Fig. 5 shows that the state estimates converge to the true values, and that the origin is stabilized using time-varying output feedback.


Fig. 5: Output feedback for the mass-spring example using the forward-in-time controller. Top: State trajectories and estimates. Bottom: Control action.

We now consider an example in which the forward-intime controller with full state measurement fails to achieve stabilization. Let

$$
A=\left[\begin{array}{ll}
1 & 0 \\
0 & 1
\end{array}\right], \quad B=\left[\begin{array}{c}
\sin (\omega t) \\
\cos (\omega t)
\end{array}\right],
$$

and $\omega=1$. As before, we first assume that $B(t)$ is known in advance and we apply the backwards-in-time optimal control (3) over a finite interval of 10 seconds. Let $R_{1}=R_{2}=I$ and $P_{\mathrm{b}}(10)=0$. Fig. $6 \mathrm{a}$ shows the state trajectories and control time history.

Next we assume that $B(t)$ is not known by the controller ahead of time. Instead, a perfect measurement of $B(t)$ is available at time $t$. We let $R_{1}=R_{2}=I, P_{\mathrm{f}}(0)=0$, and apply the forward-in-time control (10). Fig. $6 \mathrm{~b}$ shows the state trajectories and control action.

We note that, although the system is controllable and thus stabilized by the backward-in-time optimal controller, the forward-in-time controller fails. However, if we increase $\omega$ to $2 \pi \mathrm{rad} / \mathrm{sec}$ and rerun the simulation, the forward-in-time control becomes stabilizing as seen in Fig. 7. This phenomenon of stabilization via fast time variation is considered in Section IV.

Finally, we consider simultaneous stabilization of two uncoupled harmonic oscillators given by

$$
A=\left[\begin{array}{cccc}
0 & 1 & 0 & 0 \\
-1 & 0 & 0 & 0 \\
0 & 0 & 0 & 1 \\
0 & 0 & -1 & 0
\end{array}\right], \quad B=\left[\begin{array}{c}
0 \\
\sin (\omega t) \\
0 \\
\cos (\omega t)
\end{array}\right]
$$

where $\omega=2 \pi \mathrm{rad} / \mathrm{sec}$. We apply the forward-in-time control (10). Fig. 8 shows the state trajectories and control action. 


\section{NONSTANDARD RICCATI EQUATION}
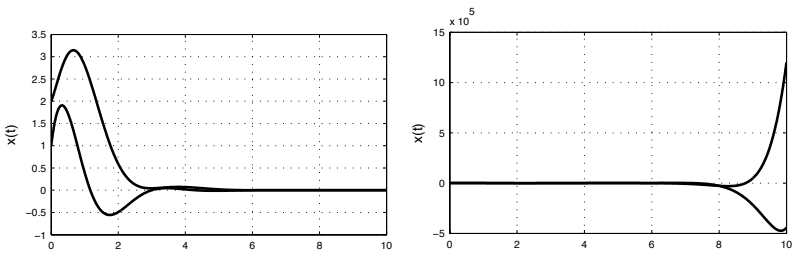

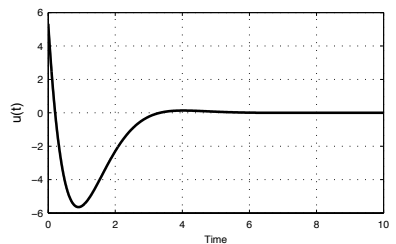

(a) Backwards-in-time control

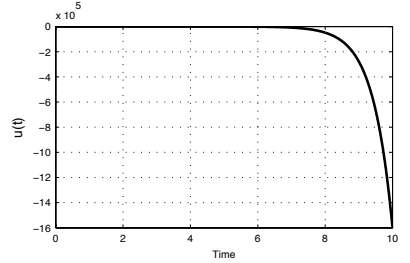

(b) Forward-in-time control
Fig. 6: Full-state feedback for $\omega=1 \mathrm{rad} / \mathrm{sec}$.
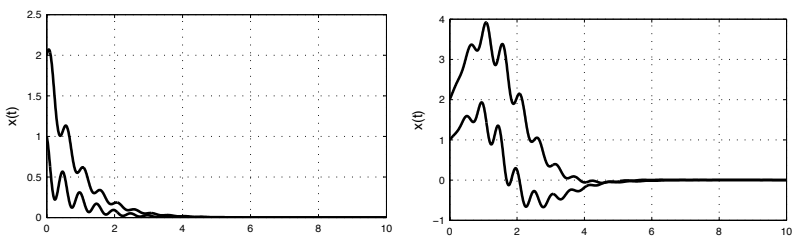

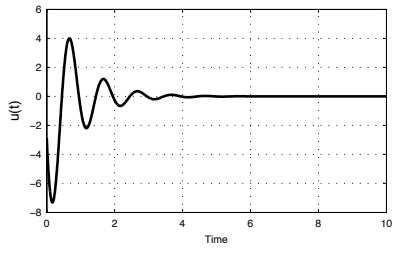

(a) Backwards-in-time control

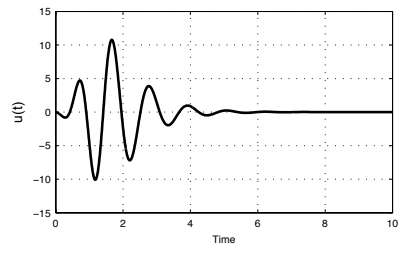

(b) Forward-in-time control
Fig. 7: Full-state feedback for $\omega=2 \pi \mathrm{rad} / \mathrm{sec}$.
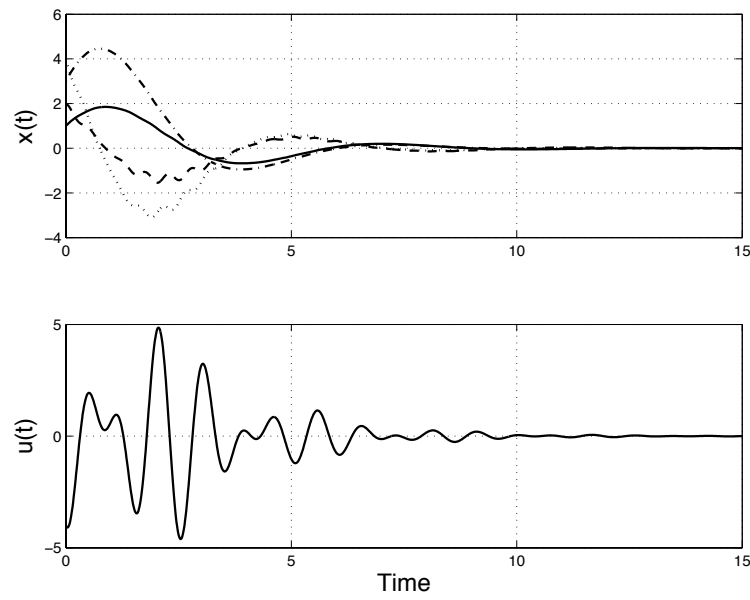

Fig. 8: Full-state feedback for the uncoupled harmonic oscillators using the forward-in-time controller. Top: State trajectories. Bottom: Control action.

\section{A. Scalar Case}

We analyze the scalar form of (1) in detail. Consider the system

$$
\dot{x}(t)=a(t) x(t)+b(t) u(t)
$$

where $a(t)$ and $b(t)$ are bounded continuous scalar functions on $[0, \infty)$. Under these assumptions, the scalar version of forward-in-time control law (10) is asymptotically stabilizing because the closed-loop scalar system is symmetric.

We now provide self-contained, alternative theorems of this result motivated by adaptive control with a slight modification and an explicit controllability condition. The proofs are omitted due to space constraints. Define

$$
\beta \triangleq \sup _{t \geq 0}|b(t)|
$$

We assume that $b(t)$ is persistently exciting, that is, there exists $T_{0}>0$ and $\varepsilon>0$ such that, for all $t \geq 0$,

$$
\int_{t}^{t+T_{0}} b^{2}(\tau) d \tau>\varepsilon
$$

Theorem 3: Let $\kappa>0$, let $\alpha_{0}>\max \{0, a(t)\}$ for all $t$, let $p$ satisfy

$$
\dot{p}(t)=2 \alpha_{0} p(t)-2 b^{2}(t) \kappa p^{2}(t),
$$

let $p(0)>0$, and let $T \geq \max \left\{T_{0}, \frac{1}{2 \alpha_{0}} \ln (2 \kappa \varepsilon p(0))\right\}$. Then, $p(t)$ exists on $[0, \infty)$ and satisfies

$$
0<p(t) \leq p_{\max }, \quad t \geq 0,
$$

where

$$
p_{\max } \triangleq \frac{1}{2 \kappa \varepsilon e^{-2 \alpha_{0} T}} .
$$

Theorem 4: Let the assumptions of Theorem 3 hold. Then

$$
V(x, t) \triangleq \frac{1}{2 p(t)} x^{2}
$$

is positive definite; that is, $V$ is nonnegative, and $V=0$ if and only if $x=0$.

Theorem 5: Let the assumptions of Theorems 3 and 4 hold and let

$$
u(t)=-\kappa p(t) b(t) x(t) .
$$

Then, $x(t) \rightarrow 0$ exponentially as $t \rightarrow \infty$.

We now present a numerical example that is related to control over an unreliable communication channel. Let $a=$ $\kappa=p(0)=x(0)=1, \alpha_{0}=2, b(t)=\max \{\sin (10 t), 0\}$. Note that the dynamics are unstable when $b(t)=0$. Fig. 9 shows the state trajectory and control action. The state of the closed-loop system converges to the origin exponentially. 

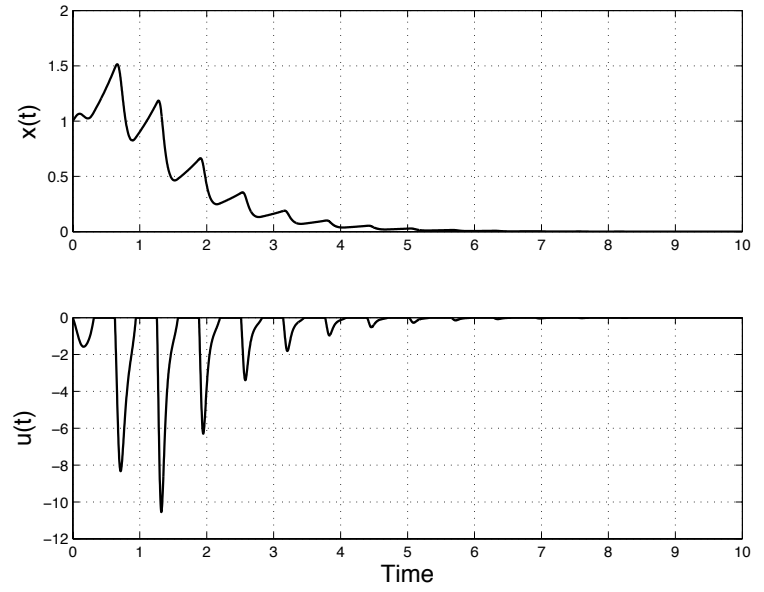

Fig. 9: Scalar Simulation

\section{B. Vector Case}

The scalar controller presented in the previous section can be generalized, under appropriate assumptions, to non-scalar systems. This generalization leads to a nonstandard Riccati equation.

Theorem 6: Let $\alpha>0$, let $P(0)>0$, and let $P$ satisfy

$$
\begin{aligned}
\dot{P}(t)= & (A(t)+\alpha I) P(t)+P(t)(A(t)+\alpha I)^{\mathrm{T}} \\
& -\Sigma(t) P^{2}(t)-P^{2}(t) \Sigma(t),
\end{aligned}
$$

where

$$
\Sigma(t)=B(t) B^{T}(t)
$$

Suppose that $P(t)$ exists on $[0, \infty)$ and satisfies

$$
0<\epsilon I \leq P(t) \leq P_{\max }, \quad t \geq 0 .
$$

Define the Lyapunov candidate

$$
V(x(t), t)=x^{T}(t) P^{-1}(t) x(t)
$$

and let

$$
u(t)=-B(t)^{T} P(t) x(t) .
$$

Then $x(t) \rightarrow 0$ exponentially as $t \rightarrow \infty$.

\section{CONCLUSION}

We have presented forward-in-time Riccati-based controllers for linear time-varying systems. The controllers do not require advance knowledge of the system dynamics and as such can be implemented in real time applications in which the system matrices are known at the present time but not in advance. Further, we have improved upon the results of [6], [7] in that we do not require a state transformation nor knowledge of the full state and, therefore, can utilize output-feedback.

We have shown that if the closed-loop dynamics of the quasi-dual system are symmetric, then the forward-in-time controller is asymptotically stabilizing. We have also shown, using averaging theory, that there exists a sufficiently high frequency for which the dynamics of the closed-loop system become asymptotically stable.
In presenting the scalar controller and the controller for the multi-input/multi-state case based on the nonstandard Riccati equation, we addressed the stabilizability of systems with persistently exciting input matrices. Consider the system

$$
\dot{x}=A x+B(t) u,
$$

where $A \in \mathbb{R}^{n \times n}$ is a constant unstable matrix, $B(t) \in$ $\mathbb{R}^{n \times m}$ is a bounded continuous function (and perhaps rank deficient). $B(t)$ is assumed persistently exciting, that is, there exists $T>0$, and $\epsilon>0$, such that for all $t \geq 0$,

$$
\int_{t}^{t+T} B(\tau) B^{\mathrm{T}}(\tau) \delta \tau>\epsilon I .
$$

As discussed in [12], stabilizability of this system is an open problem. In the present paper we answer this question positively in the special cases defined by our assumptions for multi-input systems. We note that [13] addresses the case of single-input systems using a 'persistence filter' that takes an equivalent form to the scalar Riccati equation presented in this paper.

Future work will focus on the application of the forwardin-time controller to magnetically actuated spacecraft, where the local magnetic field is measured on board the spacecraft but is not known accurately in advance. We note that the problem of inertial pointing using magnetic actuators is addressed in [14] employing averaging arguments, although the controller is not based on Riccati equations.

\section{REFERENCES}

[1] H. Kwakernaak and R. Sivan, Linear Optimal Control Systems. New York N.Y.: J. Wiley and Sons, 1972.

[2] J. Maciejowski, Predictive Control with Constraints. Harlow, England: Prentice Hall, 2002.

[3] E. Camacho and C. Bordons, Model Predictive Control, 2nd ed., Advanted Textbooks in Control and Signal Processing. Springer, 2004.

[4] W. H. Kwon and S. H. Han, Receding Horizon Control: Model Predictive Control for State Models, 1st ed. Springer, 2005.

[5] W. Kwon and A. Pearson, "A modified quadratic cost problem and feedback stabilization of a linear system," Automatic Control, IEEE Transactions on, vol. 22, no. 5, pp. 838 - 842, Oct. 1977.

[6] M. S. Chen and Y. R. Huang, "Linear time-varying system control based on the inversion transformation," Automatica, vol. 33, no. 4, pp. $683-688,1997$.

[7] M.-S. Chen and C.-Y. Kao, "Control of linear time-varying systems using forward riccati equation," Journal of Dynamic Systems, Measurement, and Control, vol. 119, no. 3, pp. 536-540, 1997.

[8] W. J. Rugh, Linear System Theory, 2nd ed. Upper Saddle River, NJ, USA: Prentice-Hall, Inc., 1996.

[9] V. Afanasiev, V. Kolmanovskii, and V. Nosov, Mathematical Theory of Control Systems Design. Kluwer, 1996.

[10] F. Callier, J. Winkin, and J. Willems, "On the exponential convergence of the time-invariant matrix Riccati differential equation," in Decision and Control, 1992., Proceedings of the 31st IEEE Conference on, 1992, pp. 1536-1537 vol.2.

[11] H. K. Khalil, Nonlinear Systems, 2nd ed. Prentice Hall, 1995.

[12] A. Loria, A. Chaillet, G. Besancon, and Y. Chitour, "On the pe stabilization of time-varying systems: open questions and preliminary answers," 44th IEEE Conference on Decision and Control, pp. 6847 - 6852, December 2005.

[13] S. Srikant and M. Akella, "Persistence filter-based control for systems with time-varying control gains," Systems \& Control Letters, vol. 58, no. 6, pp. 413 - 420, 2009.

[14] M. Lovera and A. Astolfi, "Spacecraft attitude control using magnetic actuators," Automatica, vol. 40, no. 8, pp. 1405 - 1414, 2004. 